\title{
EDAFOLOGÍA, PALINOLOGÍA Y ANTROPOLOGÍA FÍSICA APLICADAS A LA ARQUEOLOGÍA AMBIENTAL
}

\author{
NOEMÍ SILVA-SÁNCHEZ ${ }^{(1)}$, OLALLA LÓPEZ-COSTAS ${ }^{(2)(3)} \&$ REBECA TALLÓN-ARMADA $^{(1)}$
}

Resumen:

\begin{abstract}
La reconstrucción paleoambiental puede darnos información muy importante acerca de cómo las sociedades hicieron uso del medio, y de cómo, a su vez, las variaciones climáticas y ambientales han influido en el devenir de las sociedades. En este trabajo analizamos el potencial de la edafología, la palinología y la antropología física en los estudios paleoambientales aplicados a la arqueología. Además, partiendo de estas disciplinas y, centrándonos en los periodos romano y germánico, exponemos tres casos de estudio del Noroeste Ibérico: i) antropización y evolución ambiental en un sector de montaña, ii) evolución de un sector del litoral asociado a una explotación salinera y iii) estudio antropológico de dos necrópolis gallegas.
\end{abstract}

Palabras clave: reconstrucción paleoambiental, arqueología ambiental, edafología, palinología, antropología física

\begin{abstract}
Pedology, palynology and physical anthropology applied to environmental archeology
Palaeoenvironmental reconstruction provides us with crucial information about how past societies used its environment and how climate and environmental changes affected human societies. In this work, we analyze the potential of pedology, palynology and physical anthropology for palaeoenvironmental studies applied to archeology. Moreover, using these disciplines, and focusing on the Roman and Germanic periods, we present three study cases from Northwest Iberia: i) anthropization and environmental evolution in a mountain sector, ii) evolution of a coastal area associated with a salt-mine exploitation, iii) anthropological study of two Galician necropolis.
\end{abstract}

Keywords: environmental reconstruction, environmental archaeology, pedology, palynology, physical anthropology

\section{LOS ESTUDIOS PALEOAMBIENTALES EN LA ARQUEOLOGÍA}

Dentro del campo de la arqueología, la reconstrucción paleoambiental estudia los cambios en los contextos físicos y biológicos en los que el ser humano ha vivido (DINCAUZE 1987) y, si bien constituye la base de la arqueología ambiental, también podría ser considerada un aspecto de la paleoecología, ya que la distinción entre ambientes naturales y sociales es, a menudo, ambigua (DINCAUZE 2000).

La arqueología ambiental estudia las transformaciones que una sociedad dada provocó en su ambiente, así como en el impacto que los cambios ambientales tuvieron en esa sociedad, tanto si el ambiente se considera la fuerza impulsora del cambio cultural como si se interpreta como un mero factor en su desarrollo (HIRST 2013). Esta aproximación es fundamental para entender aspectos tan diversos como los cambios en el uso del terreno, la intensidad y tipo de explotación agrícola, la explotación de los recursos, el nivel de desarrollo industrial, las condiciones de vida (incluyendo aspectos de salud y dieta), la existencia de redes comerciales o aspectos relacionados con el estatus social y, por ello, debe ser una parte integral de una adecuada investigación arqueológica (AEA 1995).

En las últimas décadas, la arqueología ambiental se ha convertido en una de las aproximaciones más populares para la interpretación de los procesos prehistóricos e históricos, pues su potencial ha sido más que probado a distintas escalas temporales y espaciales.

\footnotetext{
(1) Departamento de Edafoloxía e Química Agrícola. Universidad de Santiago de Compostela. España. n.o.e.s@hotmail.com; rebeca.tallon@usc.es

${ }^{(2)}$ Laboratorio de Antropología Física. Universidad de Granada. España. olallalc@gmail.com

(3) Área de Antropoloxía Física. Universidade de Santiago de Compostela. España
} 
Respecto a la escala temporal, cabe comentar que los inicios de la arqueología ambiental estuvieron íntimamente ligados a la investigación de épocas prehistóricas ya que, unido a la imposibilidad de manejar fuentes documentales, los condicionantes ambientales de estas sociedades han sido interpretados a menudo como la única explicación razonable para entender su economía, sus asentamientos y su cultura material (SMYNTYNA 2003). Sin embargo, cada vez son más los expertos en periodos culturales históricos que comprenden la importancia de tener en cuenta la información paleoambiental en sus investigaciones. Respecto a la escala espacial, vale la pena incidir en la importancia de ampliar el objeto de estudio no sólo al yacimiento arqueológico sino también a su entorno, pues la actividad humana raramente se circunscribe de forma exclusiva al espacio representado hoy por el yacimiento. Así, en la investigación paleoambiental, la combinación yacimiento-entorno puede potencialmente ofrecer una visión más aproximada a la compleja relación de los grupos humanos con los medios que estos habitaron y de los cuales obtuvieron sus recursos (MARTíNEZ-CORTIZAS 2000).

Según Dincauze (2000), los estudios paleoambientales integran a las ciencias históricas de un modo similar al que la arqueología integra a las ciencias sociales. $\mathrm{La}$ reconstrucción paleoambiental aplicada a la arqueología, o arqueología ambiental, emplea técnicas y conceptos desarrollados en disciplinas como la biología, la ecología, la zoología, la botánica, la geología, la oceanografía, la climatología, la antropología o la edafología, entre otras. Aunque nadie puede ser un experto en todas estas disciplinas $\mathrm{y}$, por lo tanto, una característica intrínseca a este tipo de investigación es la colaboración entre expertos de distintas materias.

En el presente trabajo, analizamos el potencial de la edafología, la palinología y la antropología física para la resolución de incógnitas paleoambientales con interés para la arqueología. Además, a modo de ejemplo, presentamos tres casos de estudio en los que la aplicación de alguna de estas técnicas, en yacimientos arqueológicos o en archivos ambientales del noroeste peninsular, ha permitido obtener una valiosa información que muy difícilmente se podría haber logrado sin la aplicación de estas disciplinas.

\section{LA EDAFOLOGÍA APLICADA A LA ARQUEOLOGIA}

Los suelos se forman a partir de un material de partida (roca alterada, sedimentos o material biológico en descomposición) mediante procesos de alteración química o mecánica. Estos procesos, que varían en complejidad, dependen del material parental, de la topografía, del clima, de la vegetación y del tiempo transcurrido. A pesar de que la edafología, la ciencia encargada del estudio del suelo, es una disciplina más o menos moderna, el ser humano ha sido consciente de la importancia del suelo desde muy antiguo. El desarrollo de la agricultura y la cerámica, por ejemplo, implican ya un cierto nivel de familiarización del ser humano con el suelo, sus componentes, sus propiedades y $\mathrm{su}$ comportamiento. La primera clasificación conocida de suelos fue realizada en China hace unos 4000 años durante la dinastía Xia y registrada en el libro "Yugong" hace unos 2500 años. Ésta era una clasificación utilitarista que en base a una de las propiedades más importantes de los suelos, su color, y a algunos aspectos relacionados con la texura edáfica, establecía nueve clases de suelos. Además cada uno de estos tipos de suelos tenía diferentes subclases en función de su fertilidad (más información en GoNG et al. 2003). Aunque han sido muchos los autores que han contribuido al avance de la edafología como disciplina, de entre ellos destacan Dokuchaev (1846-1903†) y Jenny (1899-1992†), quienes establecen la relación entre los factores de formación del suelo y sus propiedades. Sin embargo, el concepto genético del suelo no se incorpora hasta que el Soil Survey Staff de Estados Unidos publica su primera clasificación de suelos (la Soil Taxonomy; SOIL SURVEY STAFF 1975).

La ocupación y utilización del suelo por parte del ser humano y el desarrollo de diversas actividades antrópicas, provocan cambios físicos y químicos en los suelos. La edafología aplicada a la arqueología permite detectar estas modificaciones y relacionarlas con las actividades que pudieron originarlas, así como analizar de qué manera afectó a las sociedades humanas determinados cambios del medio. Las primeras aproximaciones de la edafología al mundo de la arqueología acompañan a otra serie de disciplinas como la sedimentología, la estratigrafía o la geomorfología que, en su conjunto, se engloban dentro de las geociencias y que, cuando se aplicaron en relación a la arqueología, conformaron la geoarqueología (BUTZER 1975; DAVIDSON \& SHACKLEY 1976). Dentro de esta última se englobarían tanto el estudio de los procesos deposicionales y postdeposicionales que habrían afectado al yacimiento como el estudio de geoindicadores de actividad humana $o$, de cambios ambientales naturales que podrían haber afectado al ser humano, dentro o fuera del contexto arqueológico.

La edafogénesis determina qué artefactos, qué características y qué indicadores ambientales (botánicos, zoológicos y edáficos) se pierden, cuáles se preservan y cuál es el grado de preservación de los mismos. Además, los factores que intervienen en laT formación de los suelos también son un componente fundamental en los procesos deposicionales y postdeposicionales que afectan a los yacimientos (HollidAY 2004). Por 
eso, la evaluación de las propiedades edáficas es de vital importancia para la correcta interpretación del contexto arqueológico (CREEMENS \& HART 1995).

En las últimas décadas se han puesto de manifiesto las oportunidades que la edafología nos presenta para interpretar la evolución de la actividad humana. Las primeras aproximaciones en este sentido se basaron en el estudio de las variaciones espaciales de las propiedades químicas del suelo para establecer pautas de ocupación. Así, los incrementos en el contenido de elementos como $\mathrm{Ca}, \mathrm{Mg} \mathrm{y}$, sobretodo $\mathrm{P}$, se relacionaron con la presencia de depósitos de huesos, materiales de desecho u hogares (e.g. HEIDENREICH \& KONRAD 1973; GRIFFITH 1981; EDWARDS et al. 1983; KONRAD et al. 1983). Del mismo modo, el estudio de las características físicas y químicas de los suelos ha sido utilizado para reconocer prácticas de enmienda en suelos agrícolas, realizadas mediante la adición de materia orgánica que previamente había sido utilizada como cama para el ganado (e.g. CONRY 1974; KosSACK et al. 1987). Además, el potencial de la edafología para la detección de procesos de erosión inducida (e.g. BIDWELL \& HOLE 1965; BINTLIFF 1976; SCHULDENREIN 1986; HÖLzER \& HÖLZER 1998; MARTÍNEZ CORTIZAS et al. 2005), para el estudio de la evolución de la contaminación atmosférica metálica ligada al desarrollo de actividades minerometalúrgicas (e.g. SHOTYK et al. 1992; MARTÍNEZ CORTIZAS et al. 1997a, 1997b, 1999a, 2013; SERRANO et al. 2011, 2013; PONTEVEDRA-POMBAL et al. 2013), o incluso para el estudio de cambios climáticos que pudieron haber afectado a las condiciones de vida de las sociedades pasadas (e.g. MARTínEZ CORTIZAS \& COSTA CASAIS 1997; KUIJPERS et al. 1999; MARTÍNEZ CORTIZAS \& VÁZQUEZ VARELA 2002; BERGLUND 2003; ORLOVE 2005; CURRÁs et al. 2012), es de vital importancia para la correcta interpretación de los procesos de cambio prehistóricos e históricos.

En el NO de la Península Ibérica destaca como pionero el trabajo de Butzer, 1967. Sin embargo será a partir de finales de los 1980, gracias a los numerosos trabajos de Martínez Cortizas y colaboradores, cuando la aplicación de la edafología a contextos arqueológicos muestra un avance más notorio. Durante este periodo se realizan numerosos estudios tanto en yacimientos arqueológicos (LLANA RODRÍGUEZ et al. 1987, 1993; MARTÍNEZ CORTIZAS 1990, 1991; MARTÍNEZ CORTIZAS et al. 1993; MARTÍNEZ Cortizas \& MoARes Dominguez 1995, 1996; MARTÍNEZ CORTIZAS \& LLANA 1997) como en otros archivos naturales (MARTÍNEZ CORTIZAS et al. 1997a, 1997b, 1999a; MARTÍNEZ CORTIZAS \& Costa CASAIS 1997).

\section{LA PALINOLOGÍA APLICADA A LA ARQUEOLOGÍA}

El polen y las esporas son estructuras altamente adaptadas para participar en los procesos de reproducción y asegurar la continuidad de las especies vegetales. Éstas se producen en abundancia y, por ello, la mayor parte son depositadas y acumuladas en el medio. Los procesos de polinización y dispersión requieren que estas células sean especialmente resistentes. Y de hecho, el principio del análisis polínico se basa en que las paredes de los granos de polen y las esporas están cubiertas por un polímero, denominado esporopolenina, muy resistente a la mayor parte de los procesos de alteración química y física, excepto la oxidación (BROOKS \& SHAW 1978). Esto tiene importantes consecuencias ya que: primero, en medios anaerobios la preservación de los palinomorfos es prácticamente indefinida $\mathrm{y}$, segundo, se pueden usar agentes químicos agresivos para la separación y concentración de los palinomorfos del resto de componentes del sedimento o el suelo en el que fueron depositados (BENNET \& WILLIS 2001).

La palinología es la ciencia encargada del estudio de los granos de polen y las esporas producidas por plantas superiores y criptógamas (MOORE \& WeBB 1978). La ornamentación y las aperturas de su pared externa, junto con las diferencias en morfología y en tamaño, permite su diferenciación taxonómica a nivel de familia o género, $y$ en algunos casos incluso a nivel de especie. Sin embargo, las líneas de demarcación de la palinología son un tanto vagas ya que, en un sentido amplio, también contempla el estudio de otros 'palinomorfos no polínicos' producidos por algas $\mathrm{u}$ hongos, $\mathrm{o}$ incluso que en realidad pertenecen al reino animal-como restos de artrópodos o poliquetos-(NILSSON \& PRAGLOWSKI 1992). Cuando el objeto de estudio son palinomorfos fósiles, hablamos de paleopalinología, y cuando éstos son obtenidos en contextos arqueológicos de arqueopalinología.

Los palinomorfos fósiles pueden encontrarse en diversos archivos naturales, como depósitos sedimentarios (sedimentos de lagos, sedimentos aluviales, sedimentos depositados en cuevas, etc.) y edáficos (turberas, suelos coluviales o aluviales, suelos policíclicos, etc.) de diversa índole. Otros, como los depósitos de hielo estratificado o los depósitos de material fecal (guano de murciélago, heces de hiena, etc.), aunque menos abundantes, también son susceptibles de contener palinomorfos fósiles. El análisis del contenido polínico de estos depósitos es la principal técnica disponible para la determinación de los cambios ocurridos en la 
vegetación durante el Cuaternario y de su respuesta a los cambios ambientales incluida la actividad humana (BENNET \& WILLIS 2001). Además, la presencia de ciertos tipos polínicos puede considerarse evidencia del desarrollo de actividades agrícolas o ganaderas, o indicadora de las condiciones climáticas preponderantes en el pasado.

Tras los primeras descripciones morfológicas de polen y esporas fósiles obtenidas de depósitos postglaciares en el S. XIX (e.g. GÖPPERT 1836; EHRENBERG,1838; KIRCHHEIMER 1940), el primer investigador en utilizar registros de polen fósil para explicar los cambios ocurridos en la vegetación de un lugar fue el botánico sueco Langerhein (en WITTE 1905), quien además fue la primera persona en aplicar el cálculo de porcentajes al análisis paleopolínico. Sin embargo, es el exhaustivo trabajo de una serie de secuencias de turba en Suecia, defendido por Von Post en una reunión científica en Oslo en 1916, el que sentó las bases de la paleopalinología moderna.

En un principio, el análisis polínico se utilizó dentro del campo de la geología como una técnica de datación, en la que el contenido relativo de palinomorfos hacía posible la correlación de distintos estratos cuando otros métodos de correlación fallaban.

Sin embargo, el estudio de sedimentos lacustres laminados (que permiten obtener cronologías aproximadas) y la introducción de los métodos de datación propiciaron su evolución hacia un método de investigación sobre la evolución de la vegetación, el clima del Cuaternario y/o la actividad humana.

A principios de los años 1940, Iversen (1941) amplió la utilidad de la palinología para la solución de incógnitas directamente relacionadas con la arqueología al datar el inicio del periodo neolítico en Dinamarca. Siguiendo el ejemplo de Iversen, otros palinólogos comenzaron a usar datos polínicos para clarificar e interpretar los datos arqueológicos. Así, Godwin (1944a, 1944b, 1956), Mitchel (1951, 1956), Morrison (1959), TroelsSmith (1960) o Dimbleby (1960), entre otros, mostraron cómo los datos polínicos pueden ser usados para justificar cambios composicionales en los bosques debido al desarrollo de actividades agrícolas y/o ganaderas.

En España, los primeros estudios paleopalinológicos se realizaron ya en la década de 1940 (BELLOT RodrigueZ \& VieITEZ CORTIZO, 1945) sobre depósitos turbosos gallegos. En las de 1950, 1960 y 1970, gracias a los trabajos de Menéndez Amor (MENÉNDEZ AMOR \& FLORSCHÜTZ 1959, 1961a, 1961b; FLORSCHÜTZ \& MENÉNDEZ AMOR 1962; MENÉNDEZ AMOR 1975) se estudian un gran número de archivos naturales que son interpretados en clave antrópica y comienzan a hacerse estudios en contextos arqueológicos a lo largo de todo el territorio nacional. Sin embargo, no será hasta finales de la década de los 1970, y durante la de los 1980, gracias a las investigaciones de López García (1978, 1983,1984a, 1984b, 1985, 1986) y Dupré (1986, 1988), cuando la arqueopalinología se consolida en nuestro país. Centrándonos en el NO de la Península Ibérica destacan los trabajos de Aira Rodríguez (AIRA RODRÍGUEZ \& VÁzQUEZ VARELA 1985; AIRA RODRÍGUEZ \& GUITIÁN OJEA 1985-86; AIRA RODRIGUEZ et al.,1987, 1989; AIRA RODRÍGUEZ \& SAÁ OTERO 1988) van Mourik (VAN MOURIK 1986) o Ramil Rego (RAMIL 1993; RAMIL REGO \& AIRA RODRÍGUEZ 1993).

\section{LA ANTROPOLOGÍA FÍSICA APLICADA A LA ARQUEOLOGÍA}

La antropología física estudia la diversidad biológica de las poblaciones humanas actuales y pasadas, incluyendo la reconstrucción de su historia evolutiva y el estudio de sus parientes próximos vivos y fósiles (HAGEN 2009; REBATO et al. 2005). Al investigar un número tan amplio de aspectos de la vida del ser humano, esta disciplina se relaciona con muchas otras, entre las que destaca la arqueología (REBATO et al. 2005).

Es difícil convenir sobre cuándo nace la antropología. Probablemente, su origen sea muy antiguo y esté ligado a los inicios de la medicina racional. Sin embargo, la palabra antropología se usa por primera vez en el siglo XVI al abrigo de la anatomía (HUNDT 1501). La antropología física se consolidará dos siglos más tarde, cuando se asuma su característica principal: la vertiente zoológica/ biológica del ser humano (COMAS 1958). El adjetivo "física" se incluye al versar los primeros estudios sobre las características físicas visibles de los seres humanos, muchas veces promovidos por un especial interés en clasificar las razas humanas, cuestión que marcará los momentos más oscuros de esta disciplina (ver MARKS 1995). Posteriormente, al análisis de sujetos vivos se une el de los restos óseos humanos.

A finales del siglo XIX, los trabajos antropológicos en versan sobre las mediciones y variabilidad del esqueleto entre los que destacan en Europa, por su influencia, los estudios craneométricos del antropólogo francés Paul Broca (1824-1880†). Poco después, a la osteometría se une el diagnóstico de las patologías antiguas, dando lugar a la rama que, en 1913, Ruffer definiría como paleopatología (AUFDERHEIDE \& RODRÍGUEZ-MARTÍN 1998; CAMPILLO 2001).

La antropología física es una ciencia muy diversa. Según la SEAF (Sociedad Española de Antropología Física), en la actualidad existen al menos 37 líneas de estudio (CALDERON 2003). Por su relación con la arqueología destacamos: estudios de población y variación humana, 
paleodemografía, osteología, paleopatología, ADN antiguo, paleodieta, antropología dental y estudios de crecimiento. En los últimos treinta años el estudio de los restos óseos humanos procedentes de contextos arqueológicos se ha llamado bioarqueología (BUIKSTRA 1977; JURMAIN et al. 2009), aunque no existe un consenso entre los especialistas sobre si adoptar ésta denominación (para entender la evolución de la bioarqueología se recomienda BUIKSTRA \& BECK 2006). Al problema de la clasificación se une el hecho de que la línea entre los restos arqueológicos y no arqueológicos no es siempre muy clara y depende de la legislación de cada país. En el caso concreto de España, la Ley de Patrimonio Histórico determina que la antigüedad necesaria para considerar un resto humano como arqueológico varía entre 50 y 100 años (MÁRQUEZ-GRANT et al. 2011). A las habituales consideraciones de los materiales arqueológicos, en el caso de los restos humanos se añaden las éticas. No se debe olvidar que se trata de restos de personas y el debate sobre si perturbar o no enterramientos humanos (incluso re-enterrar) permanece a día de hoy más abierto que nunca (ver ejemplos en A.A. ASSOCIATION, 2009; REDFERN et al. 2011).

El estudio de un esqueleto humano puede proporcionar información importante sobre su estado de salud, dieta, estilo de vida, actividad, sexo, edad y rasgos biológicos, la cual se puede extrapolar a nivel poblacional (si contamos con una muestra adecuada) (LARSEN 2002). Al contrario de lo que popularmente se cree, el análisis de un esqueleto nos revela aspectos más relacionados con la vida del individuo que con su muerte (PARKERPEARSON 2003), lo cual incide en la importancia de dichos estudios para la arqueología. Las recientes técnicas de $\mathrm{ADN}$ e isótopos estables indican que ésta es una disciplina en constante evolución.
Ambas proporcionan información directa sobre aspectos de las sociedades pasadas. Así, por ejemplo, el análisis de paleodieta mediante isótopos estables en colágeno óseo (ver revisiones en KATZENBERG 2000; LARSEN 1997; entre otros) tiene un gran auge en la actualidad.

En España existen al menos cuatro asociaciones que incluyen campos de investigación relacionados con la arqueología (Sociedad Española de Antropologia Física o SEAF, Sociedad Española de Paleopatologia, Asociación Profesional de Bioarqueología y Asociación Española de Antropología y Odontología Forense). Los inicios de la antropología física en España están relacionados con la influencia del antropólogo francés Paul Broca (MÁRQUEZ-GRANT et al. 2011; REVERTE COMA 1991). Esta disciplina ha convivido desde el principio en estrecha relación con la arqueología y desde la segunda mitad del siglo XX, el número y calidad de las publicaciones ha ido incrementándose. En el NO de la Península, probablemente ligado a la mala conservación de los restos óseos debido a la acidez del suelo, el inicio de la antropología física aplicada a la arqueología es mucho más tardío. Los primeros estudios se realizaron en la década de 1960 por el Dr. Miguel Fusté en el yacimiento de A Lanzada (BlANCO FREIJEIRO et al. 1961, 1967).

\section{CASOS DE ESTUDIO}

Exponemos tres casos de estudio, en los que mediante la aplicación de alguna de las metodologías anteriormente expuesta resolvemos incógnitas relacionadas con la evolución del ser humano. Nos centramos en el Noroeste Ibérico durante los periodos romano y germánico. En la Figura 1 se indican las localizaciones de los distintos casos de estudio.

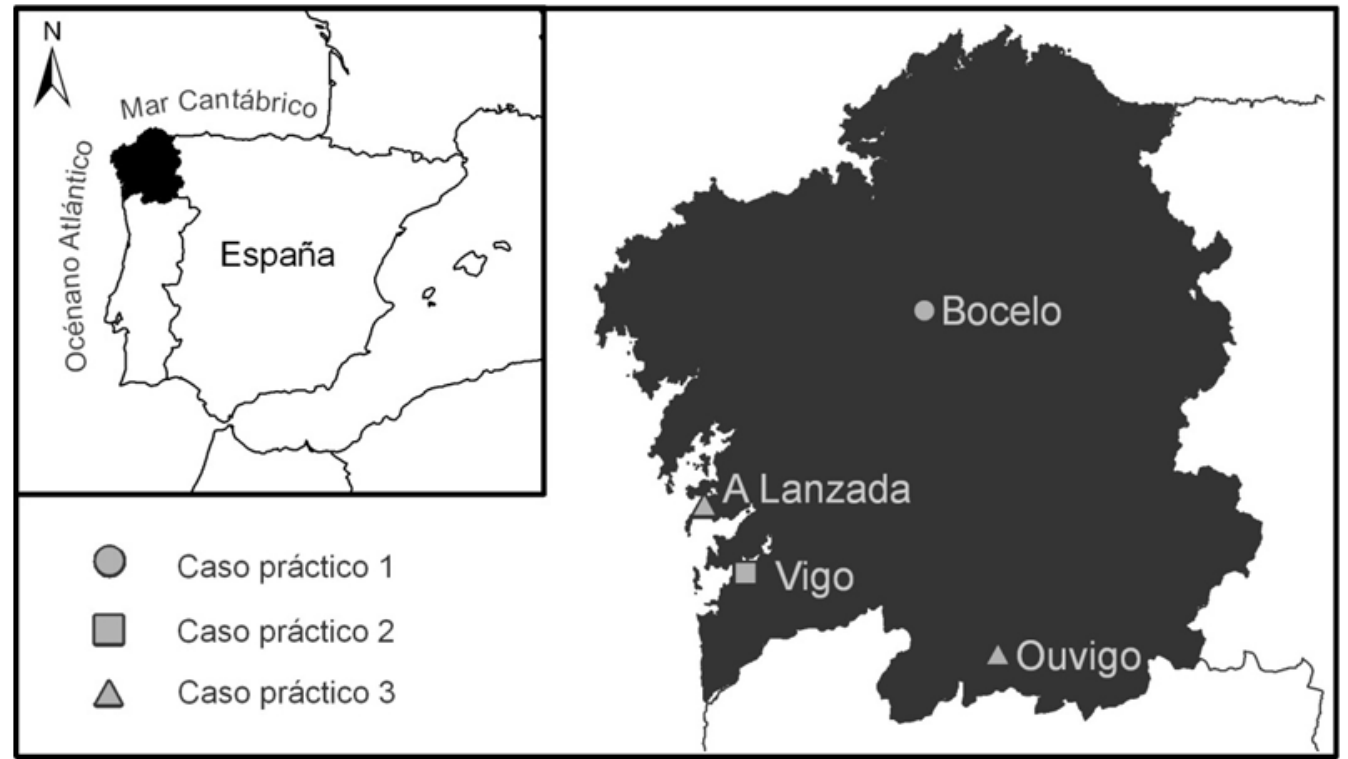

Fig. 1. Localización de los lugares en los que se realizaron los casos prácticos.

Fig. 1. Study cases locations. 


\subsection{Caso 1. Antropización y evolución ambiental en un sector de montaña}

El incremento de la presión antrópica desde la prehistoria ha sido en numerosos casos el detonante de muchos procesos de degradación ambiental. Sin embargo, para poder correlacionar adecuadamente ambos factores e identificar apropiadamente los procesos subyacentes es necesaria la aplicación de aproximaciones paleoambientales multidisciplinares. En este caso de estudio presentamos una síntesis de los resultados obtenidos mediante el análisis geoquímico y palinológico de una turbera situada en los montes de $O$ Bocelo (SILVA SÁNCHEZ 2009, 2010), para los periodos romano y germánico(Fig. 2).

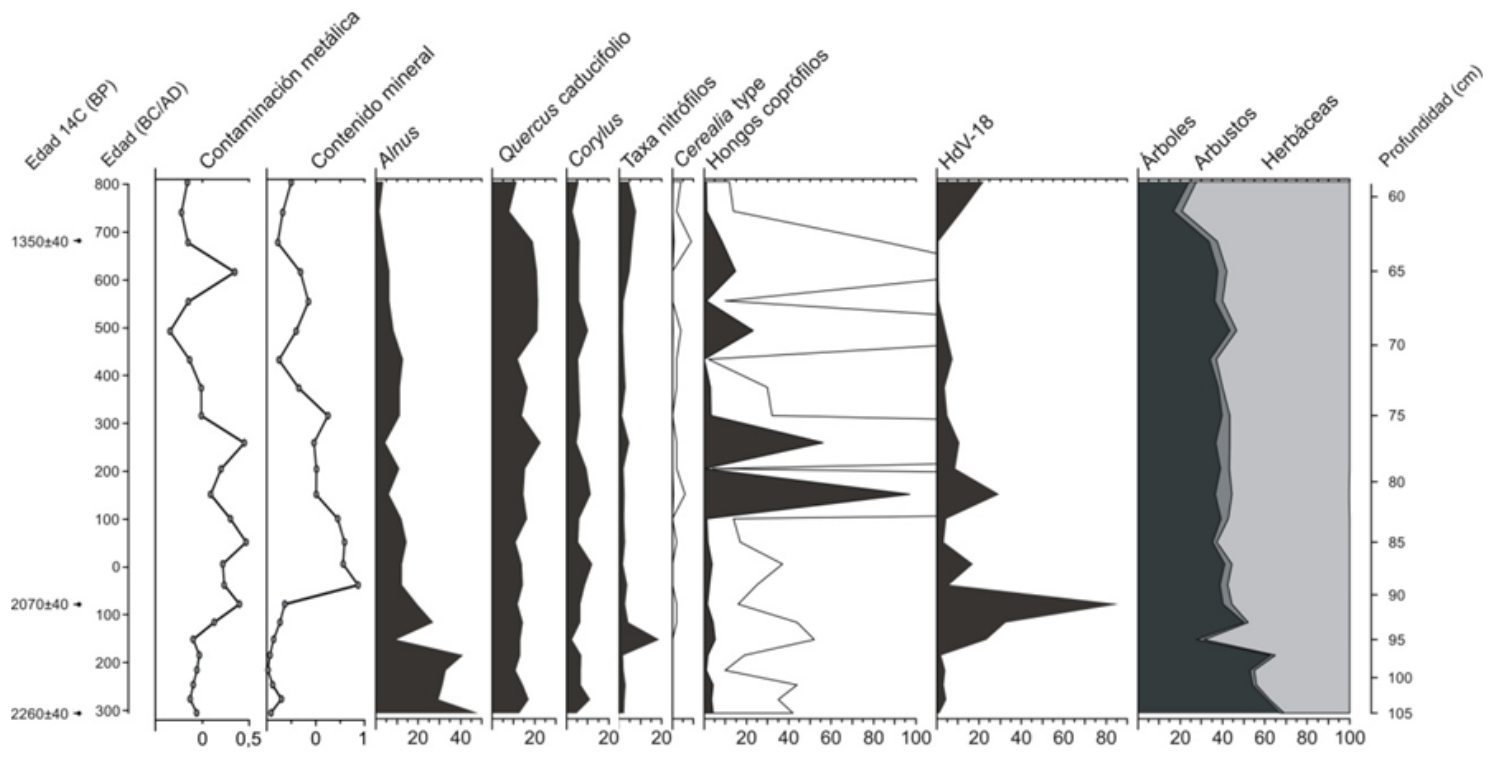

Fig. 2. Caso práctico 1. Diagrama geoquímico y palinológico sintético de la turbera Cruz do Bocelo. La contaminación atmosférica metálica y el contenido mineral están expresados en variaciones relativas (puntuaciones factoriales). Los tipos palinológicos se expresan en porcentaje.

Fig. 2. Study case 1. Geochemical and palynological synthetic diagram of the "Cruz do Bocelo" mire. Metal pollution and mineral content are expressed as relative variations (factor scores). Palynological types are expressed as percentages.

La aplicación de métodos geoquímicos para la evaluación de la paleocontaminación atmosférica metálica es una de las aproximaciones más utilizadas en la reconstrucción del desarrollo de actividades minero-metalúgicas en el pasado. En O Bocelo hemos encontrado que tanto durante el periodo romano como durante el periodo germánico se habrían producido incrementos en la contaminación atmosférica por metales. Aunque la fase de contaminación germánica es de menor duración y de menor intensidad que la romana. Coetáneamente a los incrementos de la contaminación durante el periodo romano, la señal polínica arbórea decrece debido al descenso en los valores del aliso y el avellano y, en menor medida, del roble. Aunque, al mismo tiempo, también se producen ligeros incrementos en el polen de cereal, lo que dificulta la distinción entre la influencia que las actividades minero-metalúrgicas y agrícolas tuvieron sobre los bosques. En cualquier caso, la abrupta e intensa deforestación que tuvo lugar en estos montes durante este periodo provocó a su vez una serie de cambios ambientales en cadena. Tras el episodio de deforestación, el incremento en el flujo de elementos litogénicos-marcadores de materia mineral - a la turbera indica que la intensa y abrupta deforestación supuso la erosión de los suelos de la cuenca. Además, el incremento en la presencia de un marcador polínico de condiciones húmedas (HdV-18) indicó un aumento del nivel freático de la turbera, el cual posiblemente esté ligado al descenso en la pérdida de capacidad de retención de agua de los suelos del entorno como consecuencia de la deforestación y la fuerte erosión.

Durante el periodo germánico la tendencia de reducción del bosque mixto caducifolio continúa. El buen ajuste entre los incrementos en el polen de cereal, indicador de actividades agrícolas, y en la abundancia de NPP's coprófilos, indicadores de la presencia de ganado, apuntan a que este descenso en el nivel de cobertura arbórea pudo estar fundamentalmente acoplado a la creación de campos de cultivo y pastos para el ganado. Esta deforestación, al igual que como había ocurrido durante el periodo romano, parece estar detrás del nuevo repunte de los procesos erosivos en la zona y el ascenso del nivel freático de la turbera. 
El área de O Bocelo ha sido objeto de intensas intervenciones arqueológicas (ver CRIADO BOADO 1991). Sin embargo, los yacimientos estudiados corresponden fundamentalmente a periodo prehistórico y medieval. Para los periodos romano y germánico, a pesar de las claras evidencias de impacto humano mostradas por la información paleoambiental, los restos arqueológicos son muy escasos y se restringen a unas cuantas tégulas que fueron descubiertas en el cercano valle de Furelos. Los datos aquí expuestos son un claro ejemplo de cómo la reconstrucción de los cambios ambientales a partir de archivos naturales puede proporcionar una información clave respecto al desarrollo de actividades humanas y a su impacto en el medio. Lo que a su vez permite mejorar la comprensión de los procesos de antropización en una determinada zona.

\subsection{Caso 2. Evolución de un sector del litoral asociado a una explotación salinera}

En este ejemplo presentamos la reconstrucción paleoambiental de un yacimiento situado en un sector litoral de explotación de sal en época romana, desde un enfoque edafológico y palinológico. Dicho yacimiento se encuentra situado actualmente bajo la ciudad de Vigo y se corresponde con una de las mayores estructuras salineras encontradas en Galicia, y una de las mejor conservadas del mundo romano (CASTRO CARRERA 2007).

Esta investigación se llevó a cabo a partir del estudio de las propiedades físico-químicas, la composición elemental, y el análisis estratigráfico, granulométrico, mineralógico y palinológico de varios registros edafo-sedimentarios de dicho sector arqueológico. Este tipo de registros en medios litorales presentan un alto potencial para el estudio e interpretación de los contextos arqueológicos y su entorno, ya que su formación está sujeta a procesos deposicionales o genéticos (procesos de erosión-sedimentación) y postdeposicionales o diagenéticos (edafogénesis, eventos erosivos, etc), resultado de la interacción entre la compleja dinámica marino-continental y la actividad antrópica (distintos usos y aprovechamientos del medio).

Los resultados han permitido interpretar y reconstruir los diferentes ambientes para un rango cronológico de unos 4000 años (Fig. 3) (TALLÓN ARMADA 2012), aunque en este caso nos centraremos en los periodos romano y germánico.

Las evidencias edafo-sedimentarias estudiadas ponen de manifiesto que, en este sector, durante el periodo romano las marismas se extienden en detrimento de las formaciones de playa, lo que indica una mayor interacción marinocontinental. Coetáneamente se produce la instauración de la explotación de sal. Esta actividad se podría haber iniciado hacia el siglo II $\mathrm{BC}$ y cesaría entre los siglos III y V AD. El periodo de explotación es coincidente climáticamente con la primera fase del Periodo Cálido Romano, cálida y seca (I BC-siglo III AD) (MARTínEZ CORTIZAS \& VAZQUEZ VARELA 2002), mientras que entre los siglos III-V AD, el medio se torna más húmedo, a la vez que se produce el abandono de la actividad salinera y el retorno a un ambiente de marisma. El estudio polínico apoya esta interpretación ya que en los niveles asociados a la explotación de sal la presencia de quistes de dinoflagelados y crisofíceas evidencian que el ambiente era dominantemente marino, mientras que en la fase de abandono la abundancia de granos de polen de hidro-higrófitas, Typha latifolia tipo y Cyperaceae indica la evolución hacia condiciones más salobres (LÓPEZ MERINO 2011). El estudio polínico además indica que durante el periodo romano, en el entorno del yacimiento dominaban las formaciones vegetales abiertas de herbáceas, aunque con una cierta presencia del bosque caducifolio (castaño, avellano, aliso, sauce).

A partir de este momento y coincidiendo con el comienzo del periodo germánico (Siglo V AD) en este sector se forman sistemas dunares y de lagoon a la par que el clima se vuelve frío y más seco (Periodo Frío Altomedieval, V-IX AD; MARTÍNEZ CORTIZAS et al 1999b), lo que indica una intensificación de los procesos de continentalización. En torno a los siglos VI-VII se produce la estabilización de las dunas y su edafización, desarrollándose un paleosuelo, que coincide con una pequeña fase climática cálida (VI -VII AD) detectada para Galicia (MARTínEZCORTIZAS et al 1999a). Posteriormente, este sistema será sellado a partir del siglo VII AD por una nueva fase dunar resultado de la reactivación eólica. El análisis polínico indica que durante el periodo germánico a nivel local desaparecen los indicadores de ambiente marino y humedal, mientras que a nivel regional, se produce una reducción del bosque caducifolio, que no se recuperará, moderadamente, hasta siglos $\mathrm{X}-\mathrm{XV}$ AD.

Este caso de estudio ejemplifica como la realización de trabajos de reconstrucción paleoambiental en yacimientos arqueológicos, además de contextualizar la información arqueológica, proporciona una información de gran valor sobre las condiciones ambientales asociadas, tanto al momento de desarrollo de la actividad humana, como a las fases de abandono. 


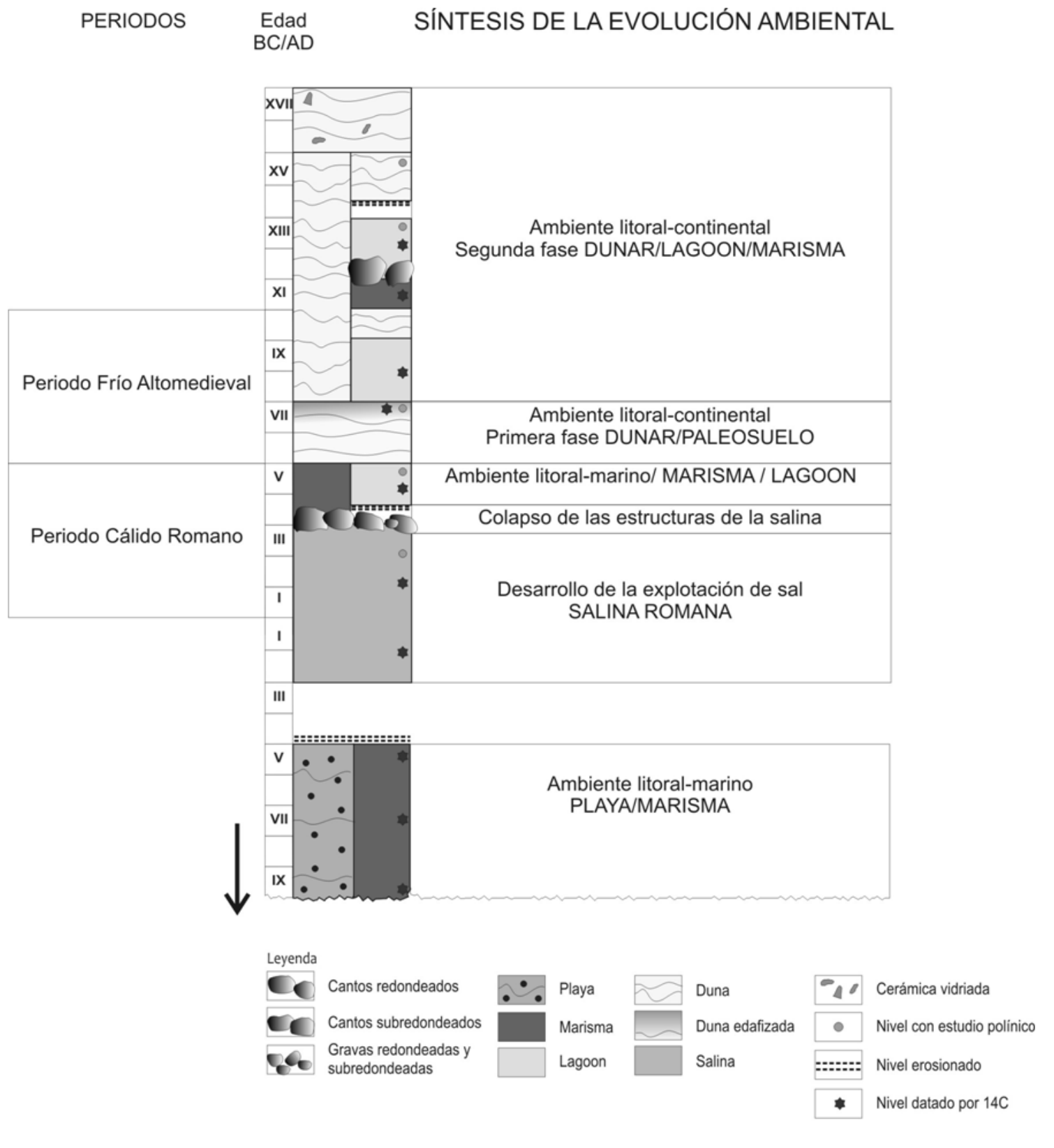

Fig 3. Caso práctico 2. Síntesis cronoestratigráfica de los registros estudiados para el sector de salinas. Relación con los eventos climáticos para los periodos romano y germánico (modificado de Tallón Armada, 2012).

Fig 3. Study case 2. Chronostratigraphic synthesis of the salt mine exploitation sector. Links with roman and germanic climate events.

\subsection{Caso 3. Estudio antropológico de dos necrópolis gallegas}

Ha resultado especialmente difícil encontrar casos de estudio en el campo de la antropología física del NO peninsular debido a la falta de yacimientos con esqueletos bien conservados. Este hecho no hace sino resaltar la importancia de cualquier análisis realizado o que se proponga en el futuro. Debido a que el tamaño muestral es un factor importante a la hora de extrapolar resultados, se presentan solo las dos necrópolis más amplias del NO (a nuestro conocimiento): A
Lanzada (85 esqueletos: 21 subadultos, $35 \hat{\jmath}, 28$, 11 adultos indeterminados) y Ouvigo (42 esqueletos: 6 subadultos, $16 \AA, 17 q, 3$ adultos indeterminados). Ambas son de excavación antigua (BLANCO FrEIJEIRO et al. 1961, 1967; CARRO OTERO et al. 1987; VARELA LÓPEZ 1985) y han sido revisadas incluyendo nuevos análisis (LÓPEZCOSTAS 2012; LOPEZ-COSTAS \& VARELA LÓPEZ, 2011). Tanto en Ouvigo como en la primera fase de A Lanzada (de periodo romano), los arqueólogos no recogieron los esqueletos infantiles, por lo que todos los subadultos conservados son juveniles (13 a 19 años), un total 
de 3 y 4 respectivamente. Resumiremos los principales hallazgos de un trabajo más amplio sobre necrópolis gallegas (LÓPEZ-COSTAS, 2012; LOPEZ-COSTAS \& VARELA LÓPEZ, 2011).
A Lanzada (Noalla, Pontevedra) es una necrópolis con dos probables momentos de uso: romano (ro) (II-IV AD) y germánico (ge) (V-VI $\mathrm{AD})$. Se trataba de una villa rural costera con una

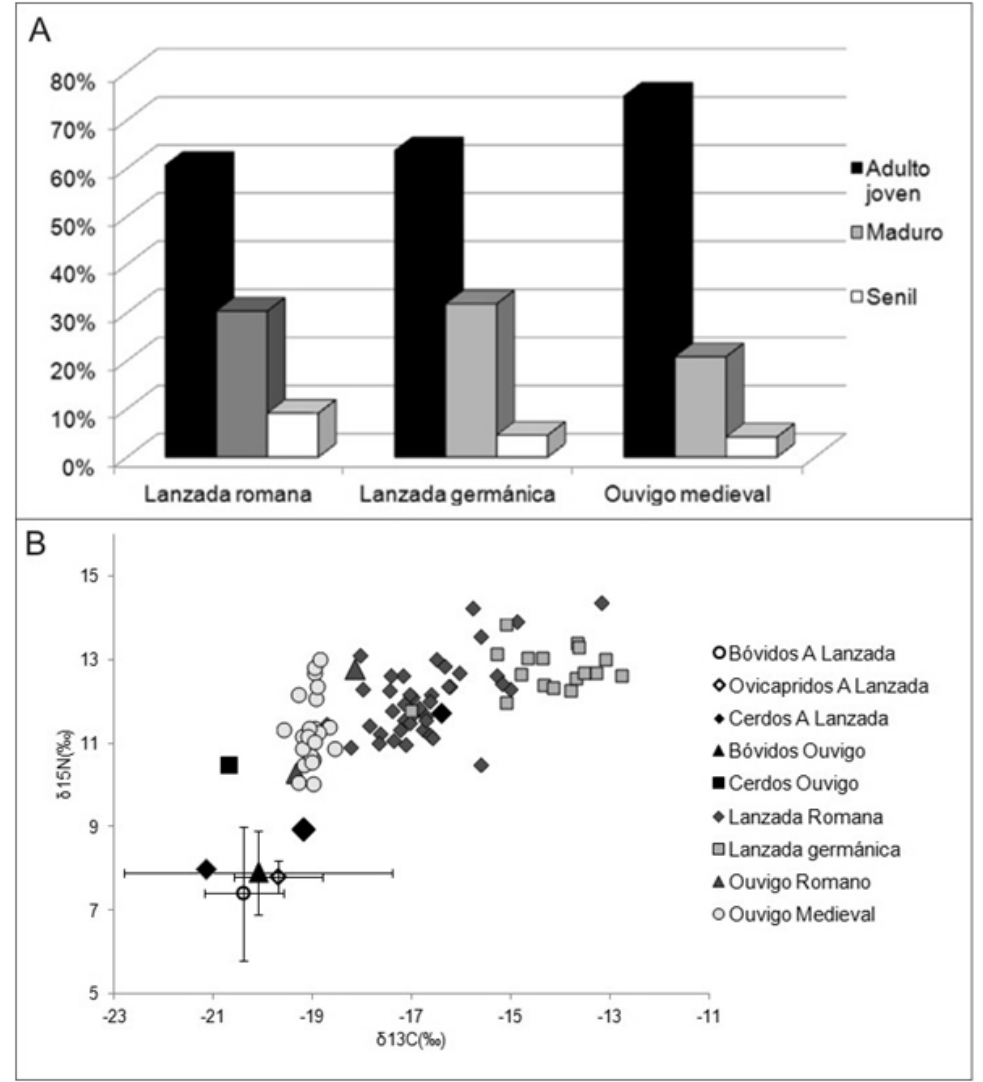

Fig. 4. Caso práctico 3. Demografía (panel A) y dieta (panel B) de A Lanzada y Ouvigo. A: gráfico de barras con los porcentajes de las series divididos siguiendo las tres categorías de edad adulta: adulto joven (20-40 años), maduro (40-60 años) y senil (>60 años); B: gráfico de dispersión con los datos isotópicos (carbono y nitrógeno) del colágeno óseo de animales y humanos, las barras de error indican la desviación típica.

Fig. 4. Study case 3. A Lanzada and Ouvigo demography (A) and diet (B). A: bar graph with adults ages: young adult (20-40 years), mature (40-60 years) and senile ( $>60$ years); B: Biplot of bone collagen from animals and humans isotopic data (carbon and nitrogen), error bars indicate the standard deviation.

posición importante en las conexiones comerciales (GONZALEZ RUIBAL 2004). El estudio paleodemográfico está marcado por el tamaño muestral limitado; teniendo en cuenta esto, la edad de muerte adulta es en ambos periodos levemente supramedia a otras necrópolis peninsulares y desciende en el promedio germánico (de 41 a 39) (LÓPEZ-CosTAs 2012). En la Figura 4 (panel A) se observa que aunque el número de adultos jóvenes

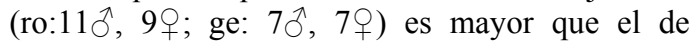
maduros (ro: $7 \hat{\jmath}, 3$; ge: $3 \hat{\jmath}, 4$ 으) y seniles (ro: $3{ }^{\lambda}$; ge: $1 \delta^{\lambda}$ ), la diferencia no es muy acusada. En cuanto a la estatura, se observa un leve dimorfismo sexual con mujeres de talla elevada con respecto a otras series peninsulares (estatura: $n=20 \hat{~}$ $165,0 \pm 4,6 / \mathrm{n}=10$ 우 157,0 $\pm 4,6$ ) (PEARSON 1899). El análisis de la dieta mediante isótopos estables revela una alimentación basada en recursos locales, sobre todo marinos y plantas C4 -probablemente mijo (López-Costas 2012). Ambos tipos se incrementan durante el periodo germánico (sobre todo el mijo), tal vez como respuesta a un deterioro ambiental (ver MARTINEZ-CORTIZAS et al. 1999a; Mighall et al. 2006) o económico.

Las tumbas de Ouvigo (Blancos, Ourense) se reparten entre el periodo Altomedieval (IX-XII $\mathrm{AD}$ ) y unas pocas que pertenecían al romano (III-V AD) (RoDríguez COLMENERO 1985). La necrópolis se construyó en el centro de una villa bastante aislada de carácter agrícola. La recolección de la muestra se limitó a cráneos y huesos largos por lo que no se pudo estimar la edad con precisión en muchos individuos. La edad de muerte adulta de la serie se sitúa entre las más 
tempranas de las medievales españolas (37 años); como se puede observar en la Figura 4 (panel B), el número de adultos jóvenes $(8 \hat{\circ}, 9+)$ es sensiblemente mayor que el de maduros $(2 \hat{\jmath}, 3+$ ) y seniles (1ㅇ). El dimorfismo sexual en la estatura es amplio, debido a la talla inframedia de las mujeres, con respecto a otras necrópolis peninsulares coetáneas (estatura: $\mathrm{n}=8 \hat{\varnothing} 166,9 \pm 3,0$ / $\mathrm{n}=8$ 153,1 $\pm 5,5)$ (PEARSON 1899). Asimismo, se observa una diferenciación social en la dieta, con un grupo minoritario con mayor ingesta de proteína animal, sin diferencias entre sexo y edades. Se ha podido detectar además presencia de mijo en la cadena alimentaria, aunque probablemente en menores cantidades que en A Lanzada (LÓPEZCOSTAS 2012).

El análisis de biodistancias (mediante un análisis cluster y discriminante en caracteres métricos y de medida media de la divergencia para no-métricos) indica que ambas poblaciones estaban muy próximas fenotípicamente, aunque la homogeneidad intrapoblacional es mayor entre los habitantes de Ouvigo, la necrópolis de la villa más aislada (LÓPEZ-COSTAS 2012; LOPEZ-COSTAS \& VARELA LÓPEZ 2011). Ambos grupos utilizaban recursos locales para su alimentación y se ha detectado presencia de mijo, ya sea como alimento humano o de animales, hecho que se ha manifestado en otras poblaciones europeas (HAKENBECK et al. 2010; LIGHTFOOT et al. 2012). Los pocos indicadores analizados sugieren que la calidad de vida podría ser menor en Ouvigo, aunque ambas poblaciones estuvieron probablemente en el límite de la supervivencia (LÓPEZ-COSTAS 2012), como sucede en la mayoría de las sociedades del periodo preantibiótico.

Finalmente, si bien el número de yacimientos y muestras analizado no es muy elevado, gracias al análisis antropológico hemos podido reconstruir aspectos relacionados con la dieta y la calidad de vida de dichas series o poblaciones.

\section{CONCLUSIONES}

Los estudios paleoambientales son propuestas reales para entender la evolución de los procesos históricos y socioeconómicos. Aquí hemos analizado diversos casos de estudio del Norte de la Península Ibérica centrándonos en los periodos romano y germánico. Sin embargo, este tipo de aproximaciones han sido también exitosas en otros ámbitos geográficos y temporales. La relación existente entre los objetivos de trabajo de paleoambientalistas, antropólogos físicos y arqueólogos es una realidad que los estudios presentados en este trabajo ejemplifican. A partir de ellos, concluimos que durante los periodos romano y germánico: 1) la presión antrópica sobre el medio provoca un descenso abrupto del bosque mixto caducifolio y la aceleración de los procesos erosivos en el área de O Bocelo; 2) se produce una progresiva continentalización del medio litoral (paso de marisma a sistemas de duna y lagoon) y un cambio en la explotación de los recursos marinos (inicio y cese de la explotación de la salina) y 3) las series de las poblaciones de A Lanzada y Ouvigo se hayan próximas fenotípicamente aunque difieren bastante en otros indicadores (estatura, edad de muerte) que sugieren que la calidad de vida era menor en Ouvigo. El estudio de la dieta muestra una alimentación basada en recursos locales y destaca la presencia de mijo en la cadena alimenticia.

Los paleoambientalistas, los antropólogos físicos y los arqueólogos llevan más de 50 años advirtiendo que la colaboración entre sus respectivas disciplinas es insuficiente. En la actualidad, el debate sobre la reconsideración de los modelos de estudio de la actividad humana continúa. A pesar de que parece sencillo prescindir de las aproximaciones paleoambientales en un momento de urgencia o de falta de presupuesto, desde este trabajo exponemos la importancia de sus resultados para el estudio no solo del yacimiento sino también de su entorno y de la sociedad que lo habitó. La falta de un trabajo paleoambiental sistemático en conjunto con la arqueología supone una gran pérdida. Por ello, esperamos que en el futuro se consideren las aportaciones paleoambientales como una pieza clave para el estudio de las sociedades pasadas.

\section{AGRADECIMIENTOS}

Los casos prácticos presentados en este trabajo han sido financiados mediante los siguientes proyectos o convenios de investigación: Caso práctico 1: CGL201020672 (Plan Nacional I+D+i, Ministerio de Ciencia e Innovación) y 10PXIB200182PR (Dirección Xeral de I+D, Xunta de Galicia); Caso práctico 2: 'Realización de análises paleoambientais e arqueométricas do Xacemento do Areal' (Dirección Xeral de Patrimonio, Consellería de Cultura e Deporte. Xunta de Galicia) y 90SECO15606PR (Consellería de Economía e Industria. Xunta de Galicia); Caso práctico 3: 'Antropoloxía dos restos óseos humanos de Galicia [...]' (Dirección Xeral de Patrimonio, Consellería de Cultura e Deporte. Xunta de Galicia). Silva Sánchez, N. está actualmente financiada con fondos del Ministerio de Educación (Programa FPU: AP2010-3264).

\section{BIBLIOGRAFÍA}

A.A. Association. 2009. Code of Ethics of the American Anthropological Association. [http:// www.aaanet.org/issues/policy-advocacy/Code-ofEthics.cfm] Retrieved 29/04/2013

AEA. 1995. Report of the Association for Environmental Archaeology Working Party on Sampling and Recovery. September 1995.

Aira Rodríguez, M.J. \& Guitián OJEA, F. 1985-86. Contribución al estudio de la Cultura Castreña gallega. Análisis palinológico de los Castros de Vixil y Penarrubia. Pontevedra Arqueológica, II: 191-200. 
AIRA RodrígueZ, M.J. \& SAÁ OTERO, M.P. 1988. Contribución al conocimiento de la vegetación holocena (3000-2210 BP) de la provincia de Pontevedra a través de análisis polínico. Anales del Jardín Botánico de Madrid, 45: 461-474.

Aira Rodríguez, M.J.; SAÁ Otero, M.P. \& DíAZ Fierros, F. 1987. Análisis polínico de un depósito periglaciar en Moucide (Lugo). Cuadernos do Laboratorio Xeolóxico de Laxe, 11: 229-137.

AIRA Rodríguez, M.J.; SAÁ OTERO, M.P. \& TABOAdA CASTRO, T. 1989. Estudios paleobotánicos y edafológicos en yacimientos arqueológicos de Galicia. Arqueoloxia/Investigación 4, Xunta de Galicia, Santiago de Compostela.

Aira Rodríguez, M.J. \& VÁzQuez VARELA, J.M. 1985. Nuevos datos palinológicos sobre la agricultura prehistórica en Galicia (España). Trabalhos de Antropologia e Etnologia., 25(2-4): 241-252.

AufDerheIDE, A.C. \& Rodríguez-Martín, C. 1998. The Cambridge encyclopedia of human paleopathology. Cambridge University Press, Cambridge.

Bellot Rodríguez, F. \& Vieitez Cortizo, E. 1945. Primeros resultados del análisis de turberas galaicas. Anales del Instituto Español de Edafología, Ecología y Fisiología Vegetal, 2: 281303

BenNet, K.D. \& Willis, J. K. 2001. Pollen. In: J.P. SMOL, H.J.B. BIRKS \& W.M. LAST (Eds.), Tracking Environmental Change Using Lake Sediments. Volume 3: Terrestrial, Algal, and Siliceous Indicators, Kluwer Academic Publishers, Dordrecht.

BERGLUND, B.E. 2003. Human impact and climate changessynchronous events and a causal link? Quaternary International, 105(1): 7-12.

BiDwELl, D.W. \& HolE, F.D. 1965. Man as a factor of soil formation. Soil science, 99: 65-72

BinTLIFF, J.L. 1976. Sediments and settlement in Southern Greece. In: D.A. DAVIDSON \& M.L. SHACKLEY (Eds.), Geoarchaeology, 267-275.

Blanco Freijeiro, A.; Fusté Ara, M.E. \& Garcia Alen, A. 1961. La necrópolis galaico-romana de $\mathrm{La}$ Lanzada (Noalla, Pontevedra), I. Cuadernos de Estudios Gallegos, 16: 141-158.

Blanco Freijeiro, A.; Fusté Ara, M.E. \& GARCIA Alen, A. 1967. La necrópolis galaico-romana de $\mathrm{La}$ Lanzada (Noalla, Pontevedra), II. Cuadernos de Estudios Gallegos, 22: 5-23, 129-155.

BRoOKS, J. \& SHAW, G. 1978. Sporopollenin: A review of its chemistry, palaeochemistry and geochemistry, Grana, 17: 91-97

BUIKSTRA, J.E. 1977. Biocultural Dimensions of Archeological Study: A Regional Perspective. In: R.L. BLAKely (Ed.), Biocultural Adaptation in Prehistoric America. Proceedings of the Southern Anthropological Society N11. University of Georgia Press, Athens: 67-84.

Buikstra, J.E. \& BECK, L.A. 2006. Bioarchaeology: The Contextual Analysis of Human Remains. Academic Press, London.

BUTZER, K.W. 1967. Geomorphology and stratigraphy of the paleolithic site of Budiño. Eiszeitalter und Gegenwart, 33: 95-117.
ButZER, K.W. 1975. The ecological approach to Archaeology: Are we really trying? American Antiquity, 40: 106-111.

CALDERON, R. 2003. La antropología física en España [http://www.seaf.es/] Retrieved 29/04/2013

CAMPILlo, D. 2001. Introducción a la paleopatología. Bellaterra arqueología. Barcelona.

Carro Otero, J.; Masa VÁzQuez, M.C. \& VAREla OGANDO, M.L. 1987. Un nuevo enterramiento de la necrópolis galaico-romana de La Lanzada (NoallaPontevedra). Pontevedra Arqueológica, 2: 229-252.

CAStro CARrera, J.C. 2007. La salina romana del yacimiento de "O Areal" Vigo, (Galicia): un complejo industrial salazonero altoimperial. In: L. LANGóstena, D. Bernal \& A. ARÉvalo (Eds.), Salsas y salazones de pescado en Occidente durante la Antigüedad, Actas del Congreso Internacional CETARIAE. British Archaeological Reports, BAR Internacional Series 1686: 355-365.

COMAS, J. 1958. Buffon, 1707-1788, precursor de la antropología física. Universidad Nacional Autónoma de México, México.

CONRY, M.J. 1974. Plaggen soils - a review of man-made soils. Soils and Fertilizers, 37: 319-326.

CREMENS, D.L. \& HART, J.P. 1995. On chronostratigraphy, pedostratigraphy, and archaeological context. In: M.E. Collins, B.J. CARTER, B.G. Gladfelter \& R.J. Southard (Eds.), Pedological Perspectives in Archeological Reserarch. Special Publication 44, Soil Science Society of America, Madison, Wisc. 15-33.

Criado BoAdo, F. (Dir.). 1991. Arqueología del Paisaje: El área Bocelo-Furelos entre los tiempos paleolíticos y medievales (Campañas de 1987, 1988 y 1989). Arqueoloxía/Investigación 6. Xunta de Galicia, Consellería de Cultura e Xuventude, Dirección Xeral do Patrimonio Histórico e Documental, Santiago de Compostela.

Currás, A.; ZAMORA, L.; ReED, J.M.; GARCíA-SOTO, E.; FERrERo, S.; ARMENGOL, X.; MEZQUITA-JoANES, F.; MARQuÉS, M.A.; RIERA, S. \& JULIÀ, R. 2012 Climate change and human impact in central Spain during Roman times: High-resolution multi-proxy analysis of a tufa lake record (Somolinos, 1280 masl). Catena, 89 (1): 31-53.

DAVIDSON, D.A. \& ShACKLEY, M.L. (Eds.). 1976 Geoarchaeology: earth science and the past. Duckworth, London.

Dimbleby, G.W. 1960. Pollen analyses of a Mesolithic site at Addington. Kent. Grana Palynologica, 4: 140148.

DiNCAUZE, D.F. 1987. Strategies for paleoenvironmental reconstruction in archaeology. Advances in Archeological Method and Theory, 11: 255-336.

DINCAUZE, D.F. 2000. Environmental archaeology: principles and practice. Cambridge University Press, Cambridge.

DUPRÉ, M. 1986. Contribution de l'analyse pollinique à la connaissance du paléoenvironnement en Espagne. L'Anthropologie, 90 (3):589-591.

DUPRÉ, M. 1988. Palinología y paleoambiente. Nuevos datos españoles. Universitat de València, Valencia. 
Edwards, K.J., Hamond, F.W. \& Simms, A. 1983. The medieval settlement of Newcastle Lyons, County Dublin: an interdisciplinary approach. Proceedings of the Royal Irish Academy, 83C: 351-376.

EHRENBERG, C.G. 1838. Beobachtungen über neue Lagen fossiler Infusorien und das Vorkommen von Fichten blüten neben deutlichem Fichen holz, Haifischzahnen, Echinioiden und Infusorien in Volhynischen Feuersteine der Kreide. Monatsber. Berliner Akad.

FLORSCHÜTZ, F. \& MENÉNDEZ AMOR, J. 1962. Beitrag zur Kenntnis der quartäre vegetations geschichte Nordspaniens. Veroffentlichungen des Geobotanischen Institutes ETK Suftung Rabel, 37: 68-73.

GoDwIN, H. 1944a. Neolithic forest clearance. Nature, 153 511-512.

GodwIN, H. 1944b. Studies of the postglacial history of British vegetation. Philosophical Transactions Bulletin, 233: 275.

GoDwIN, H. 1956. The history of the Brithish flora. Cambridge University Press, London/New York.

Gong, Z., Zhang, X., Chen, J. \& Zhang, G. 2003. Origin and development of soil science in ancient China. Geoderma 115: 3-13.

Gonzalez Ruibal, A. 2004. Facing two seas: Mediterranean and Atlantic contacts in the NorthWest of Iberia in the first millenium BC. Oxford Journal of Archaeology, 23 (3): 287-317.

GÖPPERT, H.R. 1836. De floribus in statu fossilis commentatio. Nova Acta Academiae Caesareae Leopoldino-Carolinae Germanicae Naturae Curiosorum, 18 (I): 547-572.

GRIFFITH, M.A. 1981. A pedological investigation of an archaeological site in Ontario, Canada. II. Use of chemical data to discriminate features of the Benson site. Geoderma, 25: 27-34.

HAGEN, E. 2009. American Association of Physical Anthropology: welcome to AAPA. [http:/ www.physanth.org/] Retrieved 30/04/2013

Hakenbeck, S., McManus, E., Geisler, H., Grupe, G. \& O'CONNELL, T.C. 2010. Diet and mobility in early medieval Bavaria: A study of carbon and nitrogen stable isotopes. American Journal of Physical Anthropology, 143 (2): 235-249.

HeIDENREICH, C.E. \& KonRAD, V.A. 1973. Soil analysis at the Robitaille site. Part II. A method useful in determining the location of longhouse patterns. Ontario Archaeology, 20: 33- 62.

HIRST, K.K. 2013. Environmental archaeology. [www.archaeology.about.com]. Retrieved 2013.

Holliday, V.T. 2004. Soils in archeological research. Oxford University Press, New York.

Hölzer, A. \& Hölzer, A. 1998. Silicon and titanium in peat profiles as indicators of human impact. The Holocene, 8: 685-696.

HUNDT, M. 1501. Antropologium de hominis dignitate, natura et proprietatibus, de elementis, partibus et membris humani corporis [...]. Wolfgang Stöckel, Leipzig.

IVERSEN, J. 1941. Land occupation in Denmark's stoneage. Danmarks Geologiske Undersogelse, 2 (66): 1-67.
JuRmain, R.; Kilgore, L. \& Trevathan, W. 2009. Essentials of Physical Antropology. Wadworth Cengage Learning, Belmont. $8^{\mathrm{a}}$ edición.

KATZENBERG, M.A. 2000. Stable isotope analysis: a tool for studing past diet, demography, and life history. In KATZENBERG, M.A. \& R. SAUNDERS, S. (Eds.), Biological anthropology of the human skeleton. Wiley-Liss, New Jersey: 413-441.

KIRChHEIMER, F. 1940. Hundert Jahre Pollen for schungim Dienste der Paläobotanik. Planta, 31: 414.

KonRAD, V.A.; BonNischen, R.R. \& CLAY, V. 1983. Soil chemical identification of ten thousand years of prehistoric human activity areas at the Munsungen Lake thoroughfare, Maine. Journal of Archaeological Science, 10: 13-28.

KosSACK, D.; AVERDIECK, F-R.; Blume, H-P.; HARCK, O.; HofFManN, D.; Kroll, H.I. \& ReIChsteM, J. 1987. Archsum auf Sylt - Landwirtschaft und Umwelt in vor- und frühgeschichtlicher Zeit.Ph. v. Zabern, Mainz.

KuiJPers, A.; ABRAHAMSEN, N.; HofFmANN, G.; HÜHNERBACH, V.; KONRADI, P.; KUNZENDORF, H.; MikKelsen, N.; Thiede, J. \& WeinReBe, W. 1999. Climate change and the Viking-age fjord environment of the Eastern Settlement, South Greenland. Geology of Greenland Survey Bulletin, 183: $61-67$.

LARSEN, C.S. 1997. Bioarchaeology: Interpreting behavior from the human skeleton. Cambridge University Press, Cambridge.

LARSEN, C.S. 2002. Bioarchaeology: The Lives and Lifestyles of Past People. Journal of Archaeological Research, 10 (2): 119-166.

Lightfoot, E.; Šlaus, M. \& O'Connell, T.C. 2012. Changing cultures, changing cuisines: Cultural transitions and dietary change in iron age, roman, and early medieval Croatia. American Journal of Physical Anthropology, 148 (4): 543-556.

Llana Rodríguez, C; MARTínez CoRTIZAS, A. \& RAMIL REGO, P. 1993. Contribución al conocimiento de los procesos postdeposicionales en yacimientos al aire libre con secuencia edáfica en Galicia. Arqueología Espacial, 16-17: 95-104.

LlanA, C.; Soto, M.J.; Cortizas, A.M. \& LomBA, X.M. 1987. Intervención arqueológica en el yacimiento paleolítico de la cueva de A Valiña (CastroverdeLugo). Algunos aspectos metodológicos. Furada (Revista de los espeleólogos gallegos), 1: 43-46.

LÓPEZ GARcíA, P. 1978. Resultados polínicos del Holoceno en la Península Ibérica. Trabajos de Prehistoria, 35: $1-44$.

LÓPEZ GARCÍA, P. 1983. Polen para arqueólogos. Revista de Arqueología, 21: 8-13.

LÓPEZ GARcíA, P. 1984a. Análisis polínico. Estudio del yacimiento Ibérico de Castellones de Ceal (Jaén). Arqueologia Espacial, 4: 237-239.

LÓPEZ GARCÍA, P. 1984b. Análisis polínico del yacimiento de los Tolmos de Caracena (Soria). In: JIMENO, A. (Ed.). Los Tolmos de Caracena (Soria). Campañas de 1977, 1978 y 1979. Nuevas bases para el estudio de la Edad del Bronce en la zona del Alto Duero. Excavaciones Arqueológicas en España, 134. Ministerio de Cultura, Madrid: 337-338. 
LÓPEZ GARCíA, P. 1985. Resultados de análisis polínicos del Holoceno en la Meseta Española procedentes de yacimientos arqueológicos. Anales de la Asociación de Palinólogos de Lengua Española, 2: 283-288.

LÓPEZ GARCíA, P. 1986. Estudio palinológico del Holoceno español a través del análisis de yacimientos arqueológicos. Trabajos de Prehistoria, 43: 143158.

LóPeZ Merino, L. 2011. Estudio palinológico de dos depósitos (P3-6-IA y P3-6-II) localizados en una salina de época romana (Vigo, Pontevedra). Informe inédito.

LÓPEZ-Costas, O. 2012. Antropología de los restos óseos humanos de Galicia: estudio de la población romano y medieval gallega. Tesis Doctoral, Universidad de Granada.

Lopez-Costas, O. \& VARela LóPez, T.A. 2011. Estudio craneométrico comparativo de los esqueletos de la necrópolis romana de A Lanzada, Noalla, Pontevedra. El Museo de Pontevedra, 62, 2008: 2948 .

MARKS, J.M. 1995. Human biodiversity: genes, race, and history. Aldine de Gruyter, New York.

Márquez-Grant, N.; Rissech, C., Lopez-Costas, O., Alemán, I. \& CARO DOBÓN, L. 2011. Spain/ España. In: N. MÁrQuEZ-GRANT, \& L. FIBIGER (Eds.), The Routledge Handbook of Archaeological Human Remains and Legislation: an international guide to laws and practice in the excavation, study and treatment of archaeological human remains, Routledge, London: 423-438.

MARTíneZ CoRTizAS, A. 1990. Val do Inferno y Xestido III (Abadín, Lugo): datos edáficos aplicados Gallaecia, 12: 65-80.

Martínez Cortizas, A. \& Costa Casais, M. 1997 Indicios de variaciones del nivel del mar en de Vigo durante los últimos 3000 años. Gallaecia 16: 2347.

Martínez Cortizas, A. \& Llana, C. 1997. Conceptos estratigráficos y edáficos en contextos tumulares. In: A. Rodríguez CASAL (Ed.), O neolítico atlántico e as orixes do megalitismo, Universidad de Santiago de Compostela, Santiago de Compostela: 73-91.

Martínez Cortizas, A. \& Moares Dominguez, C. 1995. Edafología y Arqueología. Estudio de yacimientos arqueológicos al aire libre en Galicia. Xunta de Galicia, Santiago de Compostela.

Martínez Cortizas, A. \& Moares Domínguez, C. 1996. Estratigrafías de yacimientos arquológicos gallegos: procesos formativos y postdeposicionales. In: R. FÁBREgas VAlCARCE (Ed.), Os primeiros poboadores de Galicia: o paleolítico, Ediciós do Castro, A Coruña: 193-215.

Martínez Cortizas, A. \& VÁzquez Varela, J.M. 2002. El clima en la Galicia romana: una aproximación multidisciplinar. Revista Real de la Academia Galega de Ciencias, XXI: 87-104.

MarTínez CORTIZAS, A.; LÓPEZ-MERINO, L.; BINDLER, R.; Mighall, T. \& KYlander, M. 2013. Atmospheric $\mathrm{Pb}$ pollution in $\mathrm{N}$ Iberia during the late Iron Age/ Roman times reconstructed using the highresolution record of La Molina mire (Asturias,
Spain). Journal of Paleolimnology, 50: 71-86.

Martínez Cortizas, A.; Mighall, T.; Pontevedra Pombal, X.; Nóvoa MuÑoz, J.C.; Peiteado VARela, E. \& PiÑeIro Rebolo, R. 2005. Linking changes in atmospheric dust deposition, vegetation change and human activities in northwest Spain during the last 5300 years. The Holocene, 15(5): 698-706.

Martínez Cortizas, A.; Nóvoa MuÑoz, J.C.; Pontevedra Pombal, X.; García-Rodeja, E. \& LlANA RodRíGUEZ, C. 1997a. Paleocontaminación. Evidencias de contaminación atmosférica antrópica en Galicia durante los últimos 4000 años. Gallaecia, 16: 7-22.

Martínez Cortizas, A.; Pontevedra Pombal, X.; NÓVOA MUÑOZ, J.C. \& GARCÍA RodeJA, E. 1997b. Four thousand years of Atmospheric $\mathrm{Pb}, \mathrm{Cd}$ and $\mathrm{Zn}$ deposition recorded by the ombrotrophic peat bog of PenidoVello (Northwestern Spain). Water, Air, and Soil Pollution, 100: 387-403.

Martínez Cortizas, A.; Ramil Rego, P. \& Llana RoDRíGUEZ, C. 1993. Edafología y Palinología: aplicación al estudio de yacimientos al aire libre en Galicia. Trabalhos de antropología e etnología, 33: 449-469.

Martínez Cortizas, A.; Valcárcel Díaz, M.; Pérez Alberti, A.; Castillo Rodríguez, F. \& Blanco CHAO, R. 1999b. O cambio climático e os paleoclimas cuaternarios. In: MARTínEZ CORTIZAS, A. y PÉrez Alberti, A. (Coords.), Atlas Climático de Galicia, Xunta de Galicia, Santiago de Compostela: 180-182.

Martínez Cortizas, A.M. 1991. Estudio sedimentolóxico e das propiedades químicas e físicas da cata 12-D do xacemento paleolítico na Cova da Valiña (Catroverde, Lugo). In: Cova da Valiña (Castroverde, Lugo). Arqueología. Investigación $n^{\circ} 5$, Xunta de Galicia, Santiago de Compostela: 45 54.

MARTÍNEZ-CORTIZAS, A. 2000. La reconstrucción de paleoambientes cuaternarios: ideas, ejemplos y una síntesis de la evolución del Holoceno en el NW de la Península Ibérica. Estudos do Quaternario, 3: 31-41.

Martinez-Cortizas, A.; PonteVEdra-Pombal, X.; GARCIA-RodeJA, E.; NOVOA-MuNOZ, J.C. \& SHOTYK, W. 1999a. Mercury in a Spanish peat bog: Archive of climate change and atmospheric metal deposition. Science, 284: 939-942.

MENÉNDEZ AMOR, J. 1975. Análisis paleobotánico de algunas muestras de lignitos procedentes de Puentes de Garcia Rodriguez (La Coruña). Boletín de la Real Sociedad Española de Historia Natural (Sección Geológica), 73: 121-124.

MENÉNDEZ AMOR, J. \& FLORSCHÜTZ, F. 1959. Algunas noticias sobre el ambiente en que vivió el hombre durante el gran interglaciar en dos zonas de ambas Castillas. Estudios Geológicos, 15: 277-285.

MENÉNDEZ AMOR, J. \& FlORSChÜTZ, F. 1961a. Contribución al conocimiento de la historia de la vegetación en España durante el Cuaternario. Resultado del análisis palinológico de algunas series de muestras de turba, arcilla y otros sedimentos en los alrededores de: I. Puebla de Sanabria (Zamora), II Buelna (Asturias), Vivero (Galicia) y en Levante. Estudios Geológicos, 17: 83-99. 
MENÉNDEZ-AMOR, J. \& FLORSChÜTZ, F. 1961b. La concordancia entre la composición de la vegetación durante la segunda mitad del Holoceno en la costa de Levante (Castellón de la Plana) y en la W de Mallorca. Boletín de la Real Sociedad Española de Historia Natural (Sección Geológica), 59: 97-100.

Mighall, T.M.; Martínez Cortizas, A.; Biester, H. \& TURNER, S.E. 2006. Proxy climate and vegetation changes during the last five millennia in NW Iberia: Pollen and non-pollen palynomorph data from two ombrotrophic peat bogs in the North Western Iberian Peninsula. Review of Palaeobotany and Palynology, 141 (1-2): 203-223.

Mitchel, G. F. 1951. Studies in Irish Quaternary deposits. Proceedings of the Royal Irish Academy, 53: 14.

Mitchel, G. F. 1956. Post boreal pollen diagrams from Irish raised bogs. Proceedings of the Royal Irish Academy, 57B: 185.

Moore, P.D. \& WeBB, J.A. 1978. An Illustrated Guide to Pollen Analysis. Hodder and Stoughton, London.

MORRISON M.E.S. 1959. Evidence and interpretation of Landam in the southeast of Ireland. Sartryckur Postaniska Notiser, 112: Fax 2.

Nilsson, S. \& Praglowski, J. (Eds.). 1992. Erdtman's Handbook of Palynology. 2nd edition. Munskgaard, Copenhagen.

ORLOVE, B. 2005. Human adaptation to climate change: a review of three historical cases and some genera perspectives. Environmental Science \& Policy, 8: 589-600.

PARKER-PEARSON, M. 2003. The archaeology of death and burial. The History Press.

PEARSON, K., 1899. Mathematical contribution to the theory of evolution. V. On the reconstruction of the stature of prehistoric races. Philosophical Transactions of the Royal Society, 192: 169-244.

PonteVedra-Pombal, X.; Mighall, T.; NóvOA-MuÑoz, J.; Peiteado-VARela, E.; RodrígueZ-Racedo, J.; García-Rodeja, E. \& MARTínez Cortizas, A. 2013. Five thousand years of atmospheric $\mathrm{Ni}, \mathrm{Zn}$, As, and Cd deposition recorded in bogs from NW Iberia: prehistoric and historic anthropogenic contributions. Journal of Archaeological Science, 40: 764-777

RAMIL REgO, P. \& AIRA RodríGUEZ, C. 1993. Estudio palinológico de la turbera de Sever (Lugo). Acta botánica malacitana, 18: 125-133.

RAMIL, P. 1993. Paleoethnobotánica de yacimientos arqueológicos holocenos de Galicia (N.O. Cantábrico). Munibe, 45: 165-174.

Rebato, E.; SusanNe, C. \& Chiarelli, B. (Eds.). 2005. Para comprender la antropología biológica: evolución y biología humama. Verbo Divino, Estella, Navarra.

REDFerN, R.C.; ClegG, M.; Giesen, M.; LoE, L. \& ROBERTS, C. 2011. BABAO Code of Ethics. [http:// www.babao.org.uk/index/ethics-and-standards] Retrieved 29/04/2013

REVERTE COMA, J.M. 1991. Antropología Forense. Ministerio de Justicia, Centro de Publicaciones, Madrid.
Rodríguez COLMENERO, A. 1985. Excavaciones arqueológicas en Ouvigo, Blancos (Orense). Noticiario arqueológico hispánico, 24: 263-388.

SCHULDENREIN, J. 1986. Paleoenvironment, prehistory, and accelerated slope erosion along the central Israeli coastal plain (Palmahim): A geoarchaeological case study. Geoarchaeology, 1: 61-81.

Serrano, O.; Martínez-Cortizas, A.; Mateo, M.A.; BiEsTer, H. \& BindLER, R. 2013. Millennial scale impact on the marine biogeochemical cycle of mercury from early mining on the Iberian Peninsula. Global Biogeochemical Cycles, 27: 2130 .

Serrano, O.; Mateo, M.A.; Dueñas-Bohórquez, A.; RENOM, P.; LÓPEZ-SÁEZ, J.A. \& MARTÍNEZ CORTIZAS, A. 2011. The Posidonia oceanica marine sedimentary record: A Holocene archive of heavy metal pollution. The Science of the Total Environment. 409(22): 4831-4840.

ShotyK, W.; WAyne Nesbitt, H. \& FyFe, W.S. 1992. Natural and anthropogenic enrichments of trace metals in peat profiles. International Journal of Coal Geology, 20: 49-84.

Silva SÁNCHEZ, N. 2009. Estudio de indicadores geoquímicos en un registro de turba de la Serra do Bocelo (Melide, A Coruña). Trabajo académicamente dirigido, Universidad de Santiago de Compostela

Silva SÁnchez, N. 2010. Cambios ambientales en los últimos 3000 años en el sector occidental de la Sierra del Bocelo a partir de indicadores geoquímicos y palinológicos. Tesis de Licenciatura, Universidad de Santiago de Compostela.

SMYNTYNA, O.V. 2003. The environmental approach to prehistoric studies: concepts and theories. History and Theory, 42 (42): 44-59.

SOIL SURVEY STAFF. 1975. Soil Taxonomy: a basic system of soil classification for making and interpreting soil surveys. U.S. Dept. of Agriculture. Soil Conservation Service. U.S. Government Printing Office, Washington D.C.

TALlÓN ARMADA, R. 2012. Marco evolutivo dunha explotación salineira de época romana (Vigo, Pontevedra). Tesis de licenciatura, Universidade de Santiago de Compostela.

Troels-Smith, J. 1960. The Muldbjerg dwelling place: an early Neolithic archaeological site in the Aamosen bog, west Zealand, Denmark. Smithsonian Institution Report for 1959: 577-601.

VAN MOURIK, J.M. 1986 Pollen profiles of slope deposits in the Galician area (N.W. Spain). Koninklijk Nederlands Aardrijkskundig Genootschap/Fysisch Geografisch en Bodemkundig Laboratorium van de Universiteit van Amsterdam, Amsterdam.

VARELA LÓPEZ, T.A. 1985. Estudio antropológico de los restos óseos de Ouvigo. Apéndice 1. In: A. Rodríguez COLMENERO. Excavaciones arqueológicas en Ouvigo, Blancos (Orense), Noticiario arqueológico hispánico, 24: 373-376.

WitTE, H. 1905. Stratiotes aloides L. funnen i Sveriges postglaciala aflagringar. Geologiska Föreningen I Stockholm Förhandlingar, 27: 432-451. 